\title{
BOOK THICKNESS OF PLANAR ZERO DIVISOR GRAPHS
}

\author{
THOMAS MCKENZIE AND SHANNON OVERBAY
}

\begin{abstract}
Let $R$ be a finite commutative ring with identity. We form the zero divisor graph of $R$ by taking the nonzero zero divisors as the vertices and connecting two vertices, $x$ and $y$, by an edge if and only if $x y=0$. We establish that if the zero divisor graph of a finite commutative ring with identity is planar, then the graph has a planar supergraph with a Hamiltonian cycle. We also determine the book thickness of all planar zero divisor graphs.
\end{abstract}

\section{Book THICKNESS}

The book embedding problem, discussed in [3] and [7], is a topological embedding problem in which graphs are embedded in a structure called a book. An $n$-book is formed by joining $n$ half-planes together at a common line, called the spine. In a book embedding, the vertices are ordered along the spine and edges are embedded on the pages of the book so that no two edges cross each other nor do they cross the spine. If we let $G$ be a graph, then the book thickness of $G$, denoted by $b t(G)$, is the smallest $n$ for which $G$ admits an $n$-book embedding.

If the spine of the book is reshaped into a circle, the edges on a page may be drawn as non-intersecting chords of the circle. With this view, determining book thickness of a graph is the same as determining the minimum number of colors needed to color the chords so that no two intersecting chords have the same color. Characterizations of graphs with book thickness at most two are given in [3] and [8]. Further, the book thickness of planar graphs is discussed in [9]. We will provide highlights of key theorems below.

We observe that any graph $G$ with at least one edge must have $b t(G) \geq 1$ since edges must be placed on the pages of the book. A graph $G$ is called outerplanar if the vertices of $G$ can be placed in a circle and all edges of $G$ can be drawn as non-intersecting chords of the circle. Using the circular view of a book, the following theorem of Bernhart and Kainen [3] is clear.

Theorem 1.1. Let $G$ be a graph. Then $b t(G) \leq 1$ if and only if $G$ is outerplanar. 


\section{BOOK THICKNESS OF PLANAR ZERO DIVISOR GRAPHS}

We denote the complete graph on $n$ vertices by $K_{n}$ and the complete bipartite graph with vertex sets of size $m$ and $n$ by $K_{m, n}$. An important characterization of outerplanar graphs is given by Chartrand and Harary [4].

Theorem 1.2. Let $G$ be a graph. Then $G$ is outerplanar if and only if $G$ contains no subgraph homeomorphic to $K_{2,3}$ or $K_{4}$.

Proof. See p. 36 of [4].

Since $K_{2,3}$ or $K_{4}$ are not outerplanar, the smallest possible book thickness for each of these graphs is two. Figure 1 depicts two page embeddings of these graphs. The first page lies above the spine and the second page lies below the spine. Hence, $b t\left(K_{2,3}\right)=b t\left(K_{4}\right)=2$.
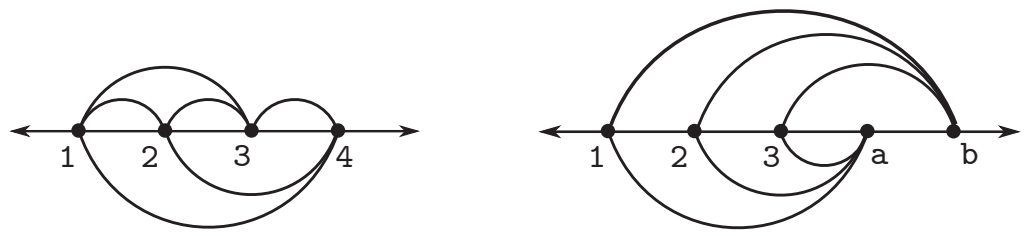

Figure 1. Two page book embeddings of $K_{4}$ and $K_{2,3}$.

Recall that a Hamiltonian cycle in a graph is a cycle that uses every vertex exactly once.

Theorem 1.3. Let $G$ be a graph. Then bt $(G) \leq 2$ if and only if $G$ is a subgraph of a planar graph with a Hamiltonian cycle.

Proof. See p. 322 of [3].

Originally, for planar graphs, it was conjectured that the book thickness was unbounded (see [3]). However, it is now known that the book thickness of planar graphs is bounded. We have the following result due to Yannakakis.

Theorem 1.4. Let $G$ be a planar graph. Then $b t(G) \leq 4$.

Proof. See [9].

The book thickness is known for several families of graphs. For others, the exact book thickness is not known. For example, although many bounds have been found, the exact book thickness of $K_{m, n}$ is unknown for arbitrary $m$ and $n$. Of course, for certain values of $m$ and $n$, the book thickness is obvious. In particular, $b t\left(K_{1, n}\right)=1$ when $n \geq 1$ and $b t\left(K_{2, n}\right)=2$ when $n \geq 3$. 


\section{T. MCKENZIE AND S. OVERBAY}

\section{Zero Divisor GraphS}

First introduced in [2], the zero divisor graph $\Gamma(R)$ of a commutative ring $R$ is formed by taking the nonzero zero divisors of $R$ as the vertices and joining two vertices with an edge if and only if the corresponding product is zero. An interesting property of these graphs is that the distance between any two vertices is quite small.

Theorem 2.1. Let $R$ be a commutative ring and let $\Gamma(R)$ be the zero divisor graph of $R$. Then $\Gamma(R)$ is a connected graph with diameter (maximum distance between two vertices) at most three.

Proof. See p. 438 [1].

Another interesting property is that two nonisomorphic rings may have isomorphic zero divisor graphs. Take, for example, the nonisomorphic rings $\mathbf{Z}_{8}$ and $\mathbf{Z}_{2}[x] /\left(x^{3}\right)$. The nonzero zero divisors in $\mathbf{Z}_{8}$ are $\{2,4,6\}$ and the corresponding zero divisor graph $\Gamma\left(\mathbf{Z}_{8}\right)$ is a path of length two. The nonzero zero divisors in $\mathbf{Z}_{2}[x] /\left(x^{3}\right)$ are $\left\{x, x^{2}, x+x^{2}\right\}$ and the corresponding zero divisor graph $\Gamma\left(\mathbf{Z}_{2}[x] /\left(x^{3}\right)\right)$ is also a path of length two.

Neal Smith [6] classified all finite rings with planar zero divisor graphs. Here, $\mathbf{F}_{q}$ denotes a finite field of order $q$, where $q$ is a prime power.

Theorem 2.2. Let $R$ be a finite commutative ring and let $\Gamma(R)$ be the zero divisor graph of $R$. Then $\Gamma(R)$ is planar if and only if $R$ is isomorphic to one of the following 44 rings.

$\mathbf{Z}_{4}, \mathbf{Z}_{2}[x] /\left(x^{2}\right), \mathbf{Z}_{9}, \mathbf{Z}_{3}[x] /\left(x^{2}\right), \mathbf{Z}_{8}, \mathbf{Z}_{2}[x] /\left(x^{3}\right), \mathbf{Z}_{4}[x] /\left(2 x, x^{2}-2\right), \mathbf{Z}_{2} \times$ $\mathbf{Z}_{4}, \mathbf{Z}_{2} \times \mathbf{Z}_{2}[x] /\left(x^{2}\right), \mathbf{Z}_{2} \times \mathbf{F}_{q}, \mathbf{F}_{4}[x] /\left(x^{2}\right), \mathbf{Z}_{4}[x] /\left(x^{2}+x+1\right), \mathbf{Z}_{4}[x] /(2, x)^{2}$, $\mathbf{Z}_{2}[x, y] /(x, y)^{2}, \mathbf{Z}_{2} \times \mathbf{Z}_{2} \times \mathbf{Z}_{2}, \mathbf{Z}_{16}, \mathbf{Z}_{2}[x] /\left(x^{4}\right), \mathbf{Z}_{4}[x] /\left(2 x, x^{3}-2\right), \mathbf{Z}_{4}[x] /\left(x^{2}-\right.$ $2), \mathbf{Z}_{4}[x] /\left(x^{2}+2 x+2\right), \mathbf{Z}_{2}[x, y] /\left(x^{2}, y^{2}\right), \mathbf{Z}_{4}[x] /\left(x^{2}\right), \mathbf{Z}_{4}[x, y] /\left(x^{2}, x y-\right.$ $\left.2, y^{2}, 2 x, 2 y\right), \mathbf{Z}_{25}, \mathbf{Z}_{5}[x] /\left(x^{2}\right), \mathbf{Z}_{3} \times \mathbf{Z}_{4}, \mathbf{Z}_{3} \times \mathbf{Z}_{2}[x] /\left(x^{2}\right), \mathbf{Z}_{2} \times \mathbf{Z}_{2} \times \mathbf{Z}_{3}$, $\mathbf{Z}_{2} \times \mathbf{Z}_{9}, \mathbf{Z}_{2} \times \mathbf{Z}_{3}[x] /\left(x^{2}\right), \mathbf{Z}_{2} \times \mathbf{Z}_{8}, \mathbf{Z}_{2} \times \mathbf{Z}_{2}[x] /\left(x^{3}\right), \mathbf{Z}_{2} \times \mathbf{Z}_{4}[x] /\left(2 x, x^{2}-2\right)$, $\mathbf{Z}_{27}, \mathbf{Z}_{3}[x] /\left(x^{3}\right), \mathbf{Z}_{9}[x] /\left(x^{2}-3,3 x\right), \mathbf{Z}_{9}[x] /\left(x^{2}-6,3 x\right), \mathbf{Z}_{8}[x] /\left(2 x-4, x^{2}\right)$, $\mathbf{Z}_{4}[x] /\left(x^{2}-2 x\right), \mathbf{Z}_{4}[x, y] /\left(x^{2}, x y-2, y^{2}-x y, 2 x, 2 y\right), \mathbf{Z}_{2}[x, y] /\left(x^{2}, y^{2}-x y\right)$, $\mathbf{Z}_{3} \times \mathbf{Z}_{9}, \mathbf{Z}_{3} \times \mathbf{Z}_{3}[x] /\left(x^{2}\right)$, or $\mathbf{Z}_{3} \times \mathbf{F}_{q}$.

Proof. See [5] and [6].

\section{Book Thickness of Planar Zero Divisor Graphs}

In this section we will classify the book thickness for each of the 44 planar zero divisor graphs from Theorem 2.2. We note that from Theorem 1.4 each of these graphs will have book thickness at most four. However, we will show that these particular graphs all have book thickness two or less. 


\section{BOOK THICKNESS OF PLANAR ZERO DIVISOR GRAPHS}

First, as we observed in Section 2, it is possible for two nonisomorphic rings to have isomorphic zero divisor graphs. In fact, although there are 44 nonisomorphic rings that correspond to planar zero divisor graphs, these only yield 18 nonisomorphic graphs. We categorize these in the following theorem.

Theorem 3.1. Let $R$ be a finite commutative ring and let $\Gamma(R)$ be the zero divisor graph of $R$. If $\Gamma(R)$ is planar, then it is one of the following 18 nonisomorphic graphs.

(1) $\Gamma\left(\mathbf{Z}_{4}\right) \simeq \Gamma\left(\mathbf{Z}_{2}[x] /\left(x^{2}\right)\right) \simeq K_{1}$

(2) $\Gamma\left(\mathbf{Z}_{9}\right) \simeq \Gamma\left(\mathbf{Z}_{3}[x] /\left(x^{2}\right)\right) \simeq K_{2}$

(3) $\Gamma\left(\mathbf{Z}_{8}\right) \simeq \Gamma\left(\mathbf{Z}_{2}[x] /\left(x^{3}\right)\right) \simeq \Gamma\left(\mathbf{Z}_{4}[x] /\left(2 x, x^{2}-2\right)\right) \simeq K_{1,2}$

(4) $\Gamma\left(\mathbf{Z}_{2} \times \mathbf{Z}_{4}\right) \simeq \Gamma\left(\mathbf{Z}_{2} \times \mathbf{Z}_{2}[x] /\left(x^{2}\right)\right) \simeq K_{1,3}$

(5) $\Gamma\left(\mathbf{Z}_{2} \times \mathbf{F}_{q}\right) \simeq K_{1, q-1}$

(6) $\Gamma\left(\mathbf{F}_{4}[x] /\left(x^{2}\right)\right) \simeq \Gamma\left(\mathbf{Z}_{4}[x] /\left(x^{2}+x+1\right)\right) \simeq \Gamma\left(\mathbf{Z}_{4}[x] /(2, x)^{2}\right)$ $\simeq \Gamma\left(\mathbf{Z}_{2}[x, y] /(x, y)^{2}\right) \simeq K_{3}$

(7) $\Gamma\left(\mathbf{Z}_{2} \times \mathbf{Z}_{2} \times \mathbf{Z}_{2}\right)$

(8) $\Gamma\left(\mathbf{Z}_{16}\right) \simeq \Gamma\left(\mathbf{Z}_{2}[x] /\left(x^{4}\right)\right) \simeq \Gamma\left(\mathbf{Z}_{4}[x] /\left(2 x, x^{3}-2\right)\right)$ $\simeq \Gamma\left(\mathbf{Z}_{4}[x] /\left(x^{2}-2\right)\right) \simeq \Gamma\left(\mathbf{Z}_{4}[x] /\left(x^{2}+2 x+2\right)\right)$

(9) $\Gamma\left(\mathbf{Z}_{2}[x, y] /\left(x^{2}, y^{2}\right)\right) \simeq \Gamma\left(\mathbf{Z}_{4}[x] /\left(x^{2}\right)\right)$ $\simeq \Gamma\left(\mathbf{Z}_{4}[x, y] /\left(x^{2}, x y-2, y^{2}, 2 x, 2 y\right)\right)$

(10) $\Gamma\left(\mathbf{Z}_{25}\right) \simeq \Gamma\left(\mathbf{Z}_{5}[x] /\left(x^{2}\right)\right) \simeq K_{4}$

(11) $\Gamma\left(\mathbf{Z}_{3} \times \mathbf{Z}_{4}\right) \simeq \Gamma\left(\mathbf{Z}_{3} \times \mathbf{Z}_{2}[x] /\left(x^{2}\right)\right)$

(12) $\Gamma\left(\mathbf{Z}_{2} \times \mathbf{Z}_{2} \times \mathbf{Z}_{3}\right)$

(13) $\Gamma\left(\mathbf{Z}_{2} \times \mathbf{Z}_{9}\right) \simeq \Gamma\left(\mathbf{Z}_{2} \times \mathbf{Z}_{3}[x] /\left(x^{2}\right)\right)$

(14) $\Gamma\left(\mathbf{Z}_{2} \times \mathbf{Z}_{8}\right) \simeq \Gamma\left(\mathbf{Z}_{2} \times \mathbf{Z}_{2}[x] /\left(x^{3}\right)\right) \simeq \Gamma\left(\mathbf{Z}_{2} \times \mathbf{Z}_{4}[x] /\left(2 x, x^{2}-2\right)\right)$

(15) $\Gamma\left(\mathbf{Z}_{27}\right) \simeq \Gamma\left(\mathbf{Z}_{3}[x] /\left(x^{3}\right)\right) \simeq \Gamma\left(\mathbf{Z}_{9}[x] /\left(x^{2}-3,3 x\right)\right) \simeq \Gamma\left(\mathbf{Z}_{9}[x] /\left(x^{2}-\right.\right.$ $6,3 x))$

(16) $\Gamma\left(\mathbf{Z}_{8}[x] /\left(2 x-4, x^{2}\right)\right) \simeq \Gamma\left(\mathbf{Z}_{4}[x] /\left(x^{2}-2 x\right)\right) \simeq \Gamma\left(\mathbf{Z}_{4}[x, y] /\left(x^{2}, x y-\right.\right.$ $\left.\left.2, y^{2}-x y, 2 x, 2 y\right)\right) \simeq \Gamma\left(\mathbf{Z}_{2}[x, y] /\left(x^{2}, y^{2}-x y\right)\right)$

(17) $\Gamma\left(\mathbf{Z}_{3} \times \mathbf{Z}_{9}\right) \simeq \Gamma\left(\mathbf{Z}_{3} \times \mathbf{Z}_{3}[x] /\left(x^{2}\right)\right)$

(18) $\Gamma\left(\mathbf{Z}_{3} \times \mathbf{F}_{q}\right) \simeq K_{2, q-1}$

Proof. Many of the details can be found in [5]. The rest are left to the reader.

Now we determine the book thickness of each of these graphs. For the next three theorems, let $R$ be a finite commutative ring with identity and let $\Gamma(R)$ be the zero divisor graph of $R$. As before, let $b t(G)$ denote the book thickness of the graph $G$.

Theorem 3.2. The book thickness of $\Gamma(R)$ is 0 if and only if $R$ is isomorphic to $\mathbf{Z}_{4}$ or $\mathbf{Z}_{2}[x] /\left(x^{2}\right)$. 


\section{T. MCKENZIE AND S. OVERBAY}

Proof. As noted in Section 1, the only graphs with book thickness zero are those comprised of isolated vertices. Clearly all such graphs are planar. In Theorem 3.1, the only planar zero divisor graphs without edges are $\Gamma\left(\mathbf{Z}_{4}\right)$ and $\Gamma\left(\mathbf{Z}_{2}[x] /\left(x^{2}\right)\right)$.

Theorem 3.3. The book thickness of $\Gamma(R)$ is 1 if $R$ is isomorphic to $\mathbf{Z}_{9}$, $\mathbf{Z}_{3}[x] /\left(x^{2}\right), \mathbf{Z}_{8}, \mathbf{Z}_{2}[x] /\left(x^{3}\right), \mathbf{Z}_{4}[x] /\left(2 x, x^{2}-2\right), \mathbf{Z}_{2} \times \mathbf{Z}_{4}, \mathbf{Z}_{2} \times \mathbf{Z}_{2}[x] /\left(x^{2}\right)$, $\mathbf{Z}_{2} \times \mathbf{F}_{q}, \mathbf{F}_{4}[x] /\left(x^{2}\right), \mathbf{Z}_{4}[x] /\left(x^{2}+x+1\right), \mathbf{Z}_{4}[x] /(2, x)^{2}, \mathbf{Z}_{2}[x, y] /(x, y)^{2}$, $\mathbf{Z}_{3} \times \mathbf{Z}_{3}, \mathbf{Z}_{2} \times \mathbf{Z}_{2} \times \mathbf{Z}_{2}, \mathbf{Z}_{16}, \mathbf{Z}_{2}[x] /\left(x^{4}\right), \mathbf{Z}_{4}[x] /\left(2 x, x^{3}-2\right), \mathbf{Z}_{4}[x] /\left(x^{2}-\right.$ $2), \mathbf{Z}_{4}[x] /\left(x^{2}+2 x+2\right), \mathbf{Z}_{2}[x, y] /\left(x^{2}, y^{2}\right), \mathbf{Z}_{4}[x] /\left(x^{2}\right)$, or $\mathbf{Z}_{4}[x, y] /\left(x^{2}, x y-\right.$ $\left.2, y^{2}, 2 x, 2 y\right)$.

Proof. The zero divisor graphs for each of these rings have at least one edge, so they must have book thickness of at least one. We see that each of the above graphs is embeddable in one page since each is a subgraph of the one page embeddable graph in Figure 2 for some $n$.

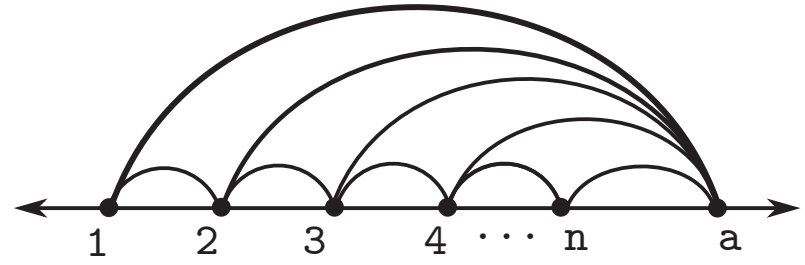

Figure 2.

Theorem 3.4. The book thickness of $\Gamma(R)$ is 2 if and only if $R$ is isomorphic to $\mathbf{Z}_{25}, \mathbf{Z}_{5}[x] /\left(x^{2}\right), \mathbf{Z}_{3} \times \mathbf{Z}_{4}, \mathbf{Z}_{3} \times \mathbf{Z}_{2}[x] /\left(x^{2}\right), \mathbf{Z}_{2} \times \mathbf{Z}_{2} \times \mathbf{Z}_{3}$, $\mathbf{Z}_{2} \times \mathbf{Z}_{9}, \mathbf{Z}_{2} \times \mathbf{Z}_{3}[x] /\left(x^{2}\right), \mathbf{Z}_{2} \times \mathbf{Z}_{8}, \mathbf{Z}_{2} \times \mathbf{Z}_{2}[x] /\left(x^{3}\right), \mathbf{Z}_{2} \times \mathbf{Z}_{4}[x] /\left(2 x, x^{2}-2\right)$, $\mathbf{Z}_{27}, \mathbf{Z}_{3}[x] /\left(x^{3}\right), \mathbf{Z}_{9}[x] /\left(x^{2}-3,3 x\right), \mathbf{Z}_{9}[x] /\left(x^{2}-6,3 x\right), \mathbf{Z}_{8}[x] /\left(2 x-4, x^{2}\right)$, $\mathbf{Z}_{4}[x] /\left(x^{2}-2 x\right), \mathbf{Z}_{4}[x, y] /\left(x^{2}, x y-2, y^{2}-x y, 2 x, 2 y\right), \mathbf{Z}_{2}[x, y] /\left(x^{2}, y^{2}-x y\right)$, $\mathbf{Z}_{3} \times \mathbf{Z}_{9}, \mathbf{Z}_{3} \times \mathbf{Z}_{3}[x] /\left(x^{2}\right)$, or $\mathbf{Z}_{3} \times \mathbf{F}_{q}$, where $q \geq 4$.

Proof. For $\mathbf{Z}_{25}$ and $\mathbf{Z}_{5}[x] /\left(x^{2}\right)$, we note that the zero divisor graph is isomorphic to $K_{4}$ and $b t\left(K_{4}\right)=2$.

For the remaining graphs, we will first demonstrate that each graph contains a $\mathbf{K}_{2,3}$ subgraph. Hence, each graph is not outerplanar and, thus, is not one page embeddable. Note that since several of these graphs are isomorphic, it is sufficient to show that it holds for one graph in each of the categories 11-18 from Theorem 3.1. Below we will demonstrate sets $A$ and $B$ which induce subgraphs containing a $K_{2,3}$.

(11) In $\mathbf{Z}_{3} \times \mathbf{Z}_{4}$, take $A=\{(1,0),(2,0)\}$ and $B=\{(0,1),(0,2),(0,3)\}$.

(12) In $\mathbf{Z}_{2} \times \mathbf{Z}_{2} \times \mathbf{Z}_{3}$, take $A=\{(0,0,1),(0,0,2)\}$ and $B=\{(0,1,0),(1,0,0),(1,1,0)\}$. 
(13) In $\mathbf{Z}_{2} \times \mathbf{Z}_{9}$, take $A=\{(0,3),(0,6)\}$ and $B=\{(1,0),(1,3),(1,6)\}$.

(14) In $\mathbf{Z}_{2} \times \mathbf{Z}_{8}$, take $A=\{(1,0),(1,4)\}$ and $B=\{(0,2),(0,4),(0,6)\}$.

(15) In $\mathbf{Z}_{27}$, take $A=\{9,18\}$ and $B=\{3,6,12\}$.

(16) In $\mathbf{Z}_{8}[x] /\left(2 x-4, x^{2}\right)$, take $A=\{x+2, x+6\}$ and $B=\{2,4,6\}$.

(17) In $\mathbf{Z}_{3} \times \mathbf{Z}_{9}$, take $A=\{(1,0),(2,0)\}$ and $B=\{(0,1),(0,2),(0,3)\}$.

(18) In $\mathbf{Z}_{3} \times \mathbf{F}_{q}$, where $q \geq 4$, take $A=\{(1,0),(2,0)\}$ and $B=$ $\{(0, a),(0, b),(0, c)\}$, where $a, b$, and $c$ are distinct units in $\mathbf{F}_{q}$.

Now we must demonstrate that two pages are sufficient to embed each of these graphs. We note that vertices of degree one do not pose any difficulty in book embeddings since they may be placed on the spine near the unique vertex to which they are adjacent and edges may be added without causing crossings (see Figure 3). The graphs in categories 11, 12 and 13 are all isomorphic to $K_{2,3}$ once we have removed all of the vertices of degree one. Hence, these graphs all have book thickness two.

For categories 14-17, see Figures 3-6 for the corresponding two page embeddings.

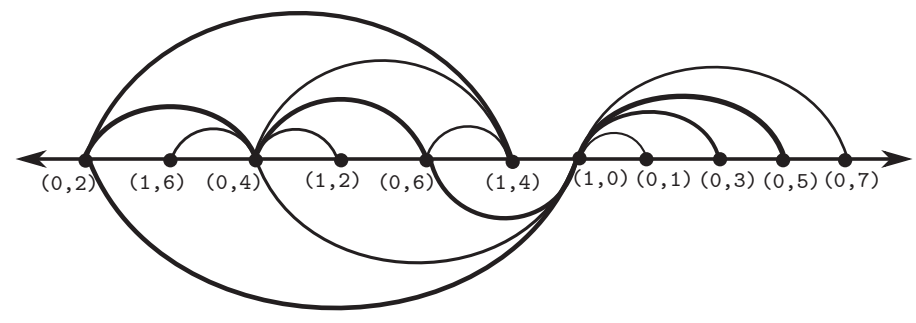

Figure 3. Two page book embedding of $\Gamma\left(\mathbf{Z}_{2} \times \mathbf{Z}_{8}\right)$.

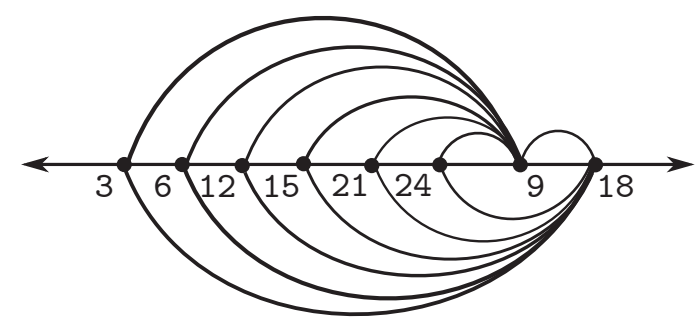

Figure 4. Two page book embedding of $\Gamma\left(\mathbf{Z}_{27}\right)$. 


\section{T. MCKENZIE AND S. OVERBAY}

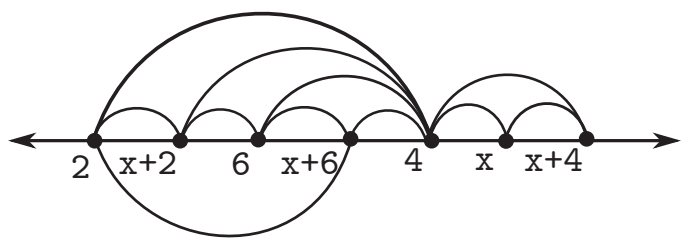

Figure 5. Two page book embedding of $\Gamma\left(\mathbf{Z}_{8}[x] /\left(2 x-4, x^{2}\right)\right)$.

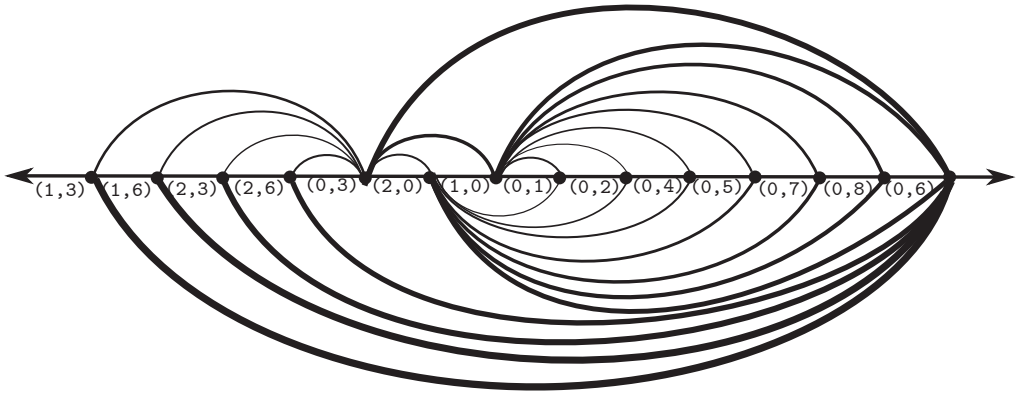

Figure 6. Two page book embedding of $\Gamma\left(\mathbf{Z}_{3} \times \mathbf{Z}_{9}\right)$.

The graph in category 18 is merely a $K_{2, q-1}$, where $q \geq 4$. As mentioned in Section 1, this graph has book thickness two.

Note that one consequence of the proof of Theorem 3.4 is that Theorem 3.3 may be strengthened to an if and only if statement since each of the graphs in Theorem 3.4 are not outerplanar. Theorems 3.2-3.4 include all 44 planar zero divisor graphs. In particular, Theorem 3.2 corresponds to category 1 of Theorem 3.1, Theorem 3.3 corresponds to categories 2-9 of Theorem 3.1, and Theorem 3.5 corresponds to categories 10-18 of Theorem 3.1. The following result is now clear.

Theorem 3.5. All planar zero divisor graphs corresponding to finite commutative rings with identity have book thickness at most two.

Combining this theorem with Theorem 1.2, we immediately obtain the following corollary.

Corollary 3.6. All planar zero divisor graphs corresponding to finite commutative rings with identity are subgraphs of planar graphs with a Hamiltonian cycle. 


\section{BOOK THICKNESS OF PLANAR ZERO DIVISOR GRAPHS}

\section{REFERENCES}

[1] D. F. Anderson and P. S. Livingston, The zero-divisor graph of a commutative ring, Journal of Algebra, 217.2 (1999), 434-447.

[2] I. Beck, Coloring of a commutative ring, Journal of Algebra, 116 (1988), 208-226.

[3] F. Bernhart and P. C. Kainen, The book thickness of a graph, Journal of Combinatorial Theory, Series B, 27.3 (1979), 320-331.

[4] G. Chartrand and F. Harary, Planar permutation graphs, Annales de l'institut Henri Poincaré (B) Probabilits et Statistiques, 3.4 (1967), 433-438.

[5] H.-J. Chiang-Hsieh, N. O. Smith, and H.-J. Wang, Commutative rings with toroidal zero-divisor graphs, Houston Journal of Mathematics, 36.1 (2010), 1-31.

[6] N. O. Smith, Planar zero-divisor graphs, International Journal of Commutative Rings, 2.4 (2003), 177-188.

[7] L. T. Ollmann, On the book thicknesses of various graphs, Proceedings of the 4th Southeastern Conference on Combinatorics, Graph Theory and Computing, 8 (1973), 459.

[8] S. Overbay, Graphs with small book thickness, Missouri Journal of Mathematical Sciences, 19.2 (2007), 121-130.

[9] M. Yannakakis, Four pages are necessary and sufficient for planar graphs, Proceedings of the Eighteenth Annual ACM Symposium on Theory of Computing, (1986), 104-108.

MSC2010: 05C10, 13M05

Key words and phrases: book thickness, zero divisor graphs, commutative rings

Department of Mathematics, Gonzaga University, Spokane, WA

E-mail address: mckenzie@gonzaga.edu

Department of Mathematics, Gonzaga University, Spokane, WA

E-mail address: overbay@gonzaga.edu 